Detection of Delayed Cerebral Ischemia (DCI) in Subarachnoid Haemorrhage Applying Near-Infrared Spectroscopy: Elimination of the Extracerebral Signal by Transcutaneous and Intraparenchymatous Measurements in Parallel

Keller, Emanuela ; Fröhlich, Jürg ; Baumann, Dirk ; Böcklin, Christoph ; Sikorski, Christopher ;

Oberle, Michael ; Muser, Markus H

DOI: https://doi.org/10.1007/978-3-319-04981-6_41

Posted at the Zurich Open Repository and Archive, University of Zurich

ZORA URL: https://doi.org/10.5167/uzh-121821

Book Section

Accepted Version

Originally published at:

Keller, Emanuela; Fröhlich, Jürg; Baumann, Dirk; Böcklin, Christoph; Sikorski, Christopher; Oberle, Michael; Muser, Markus H (2015). Detection of Delayed Cerebral Ischemia (DCI) in Subarachnoid Haemorrhage Applying Near-Infrared Spectroscopy: Elimination of the Extracerebral Signal by Transcutaneous and Intraparenchymatous Measurements in Parallel. In: Fandino, Javier; Marbacher, Serge; Fathi, AliReza; Muroi, Carl; Keller, Emanuela. Neurovascular Events After Subarachnoid Hemorrhage. Cham: Springer, 243-247.

DOI: https://doi.org/10.1007/978-3-319-04981-6_41 


\title{
Detection of Delayed Cerebral Ischemia (DCI) in Subarachnoid Haemorrhage Applying Near-Infrared Spectroscopy: Elimination of the Extracerebral Signal by Transcutaneous and Intraparenchymatous Measurements in Parallel
}

\author{
Emanuela Keller, J. Froehlich, D. Baumann, C. Böcklin, C. Sikorski, M. Oberle, and M. Muser \\ E. Keller, MD (*), C. Sikorski \\ Neurocritical Care Unit, Department of Neurosurgery, University Hospital Zurich, \\ Frauenklinikstrasse 10, Zuerich CH-8091, Switzerland \\ e-mail: emanuela.keller@usz.ch \\ C. Böcklin \\ Laboratory for Electromagnetic Fields and Microwave Electronics, ETH, Zurich, Switzerland \\ J. Froehlich, D. Baumann \\ Laboratory for Electromagnetic Fields and Microwave Electronics, ETH, Zurich, Switzerland \& NeMoDevices, Zurich, \\ Switzerland \\ M. Oberle, M. Muser NeMoDevices, Zurich, Switzerland
}

\begin{abstract}
Background: Detection of delayed cerebral ischemia (DCI) in high-grade subarachnoid haemorrhage (SAH) is an unsolved issue. Conventional near-infrared spectroscopy (NIRS) with optodes applied over the skin is controversial because the NIRS signal is contaminated by extracerebral tissue. The objective is to quantify and subtract the contribution from extracerebral tissue from the signal by using measurements in parallel with a NIRS brain tissue probe and conventional NIRS.

Methods: In a patient with high-grade SAH, two approaches for NIRS were applied. First, a conventional brain tissue probe for intracranial pressure (ICP) monitoring, supplied by optical fibres, was placed into the brain tissue $2 \mathrm{~cm}$ deep from the dura. Second, for conventional NIRS, a plaster-based patch carrying optodes (one emitter, two detectors) was attached to the skin. Central venous injections of $0.3 \mathrm{mg} / \mathrm{kg}$ body weight (bw) indocyanine green (ICG) were performed. ICG dye dilution curves obtained with the probe and patch were collected simultaneously and analysed for blood flow values.

Results: Twelve measurements in parallel with the probe and patch were performed. Mean cerebral blood flow (CBF) for the probe was higher $(24.8 \pm 9.1 \mathrm{ml} / 100 \mathrm{~g} / \mathrm{min})$ compared with the values obtained with the patch (for detector 1 , extracerebral blood flow $[\mathrm{ECBF}]$ mean $5.1 \pm 1.8 \mathrm{ml} / 100 \mathrm{~g} / \mathrm{min} ; p=0.002$; for detector $2,6.6 \pm 2.1 \mathrm{ml} / 100 \mathrm{~g} / \mathrm{min} ; p=0.002$ ). $\mathrm{CBF}$ values obtained with the probe correlated with blood flow values obtained with the patch (for CBF vs. ECBF detector 1, $r=0.72[p=0.008]$; ECBF detector 2, $r=0.79$

$[p=0.002])$.

Conclusions: Blood flow values obtained with conven- tional NIRS correlated significantly with absolute CBF values obtained directly within the brain tissue. Simultaneous measurements with the NeMo Probe and NeMo Patch allow quantification and subtraction of the contribution from extra- cerebral tissues from the signal obtained with conventional NIRS.
\end{abstract}

Keywords Subarachnoid haemorrhage, Near-infrared spectroscopy, Cerebral blood flow, Neuromonitoring 


\section{Introduction}

Detection of delayed cerebral ischemia (DCI), especially in unconscious patients with high-grade subarachnoid haemorrhage $(\mathrm{SAH})$, remains an unsolved issue. New techniques combining near-infrared spectroscopy (NIRS) and indocyanine green (ICG) dye dilution to estimate cerebral blood flow (CBF) are available [2, 10, 12]. Transcutaneous NIRS with optodes applied over the skin, however, is controversial because the NIRS signal is contaminated by extracerebral tissues (skin, skull, cerebrospinal fluid layer) $[5,7,8,11,15,16]$. To obtain measurement values directly from the brain tissue, a conventional probe for intracranial pressure (ICP) has recently been supplied with optical fibres for NIRS [9]. By measuring in parallel with the NIRS brain tissue probe and conventional NIRS through the skull, the objective is to develop algorithms to quantify and subtract the contribution from extracerebral tissues from the signal obtained by Transcranial NIRS.

Fig. 1 Two NeMo control units (NIRS instruments) are connected to the sensors NeMo Patch (plaster-based patch, strictly noninvasive approach) and $\mathrm{NeMo}$ Probe (brain tissue probe, minimally invasive approach)

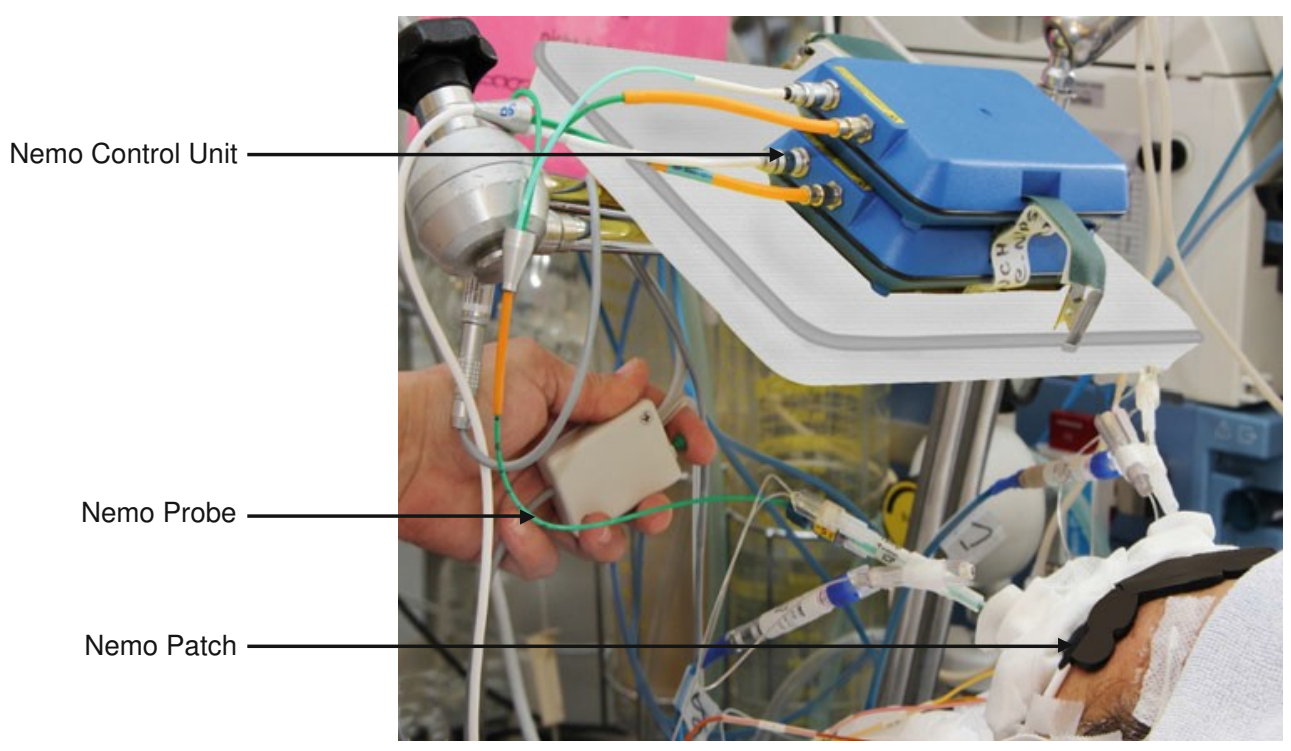

a

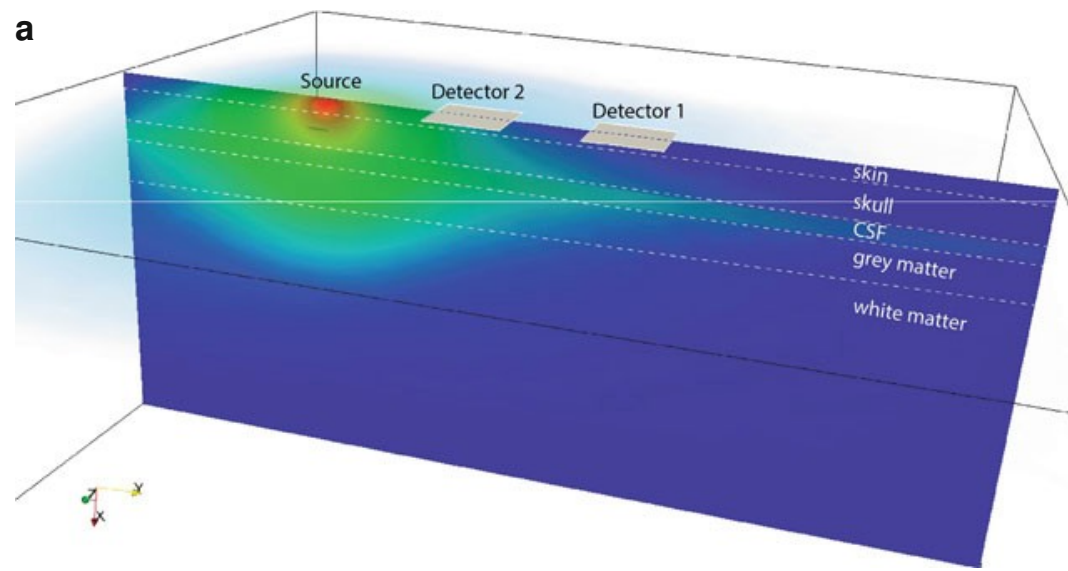

b

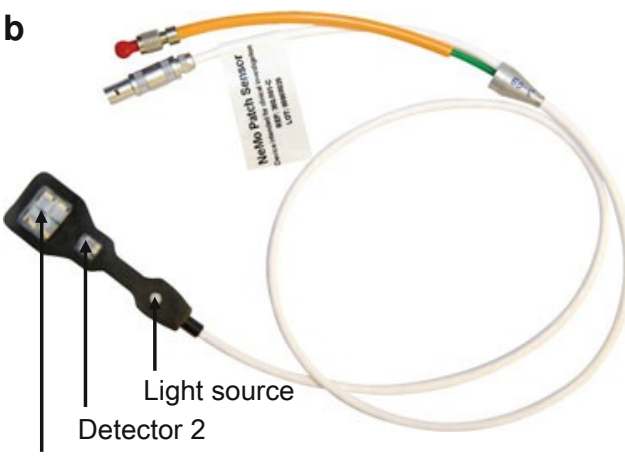

Detector 1

Fig. 2 Monte Carlo simulation of light propagation at $800 \mathrm{~nm}$ in a layered head model (a). The light intensity is shown in a logarithmic scale. Light guiding occurs in the cerebrospinal fluid layer, which has a slightly higher refractive index than the surrounding tissue. On a plaster-based patch, two detectors are placed at different distances from the laser source (detector 1, $4 \mathrm{~cm}$; detector 2, $2 \mathrm{~cm}$ away from the light source) (b 


\section{Methods}

The study was approved by the Ethics Committee of the University of Zurich. Measurements were performed in a patient with severe SAH, Hunt and Hess grade 5, Fisher grade 4, with ruptured aneurysm of the anterior communicating artery. Because of occlusive hydrocephalus, a ventricular catheter (Bactiseal, Codman; Johnson \& Johnson, USA) was inserted to drain cerebrospinal fluid. After coiling the ruptured aneurysm, the patient developed brain oedema, and ICP monitoring with an intraparenchymal probe was needed. For NIRS two approaches were applied. (1) A conventional brain tissue probe for ICP monitoring, supplied with optical fibres (NeMo Probe, NeMoDevices AG, Switzerland) was inserted through a support bolt (Integra Life Sciences, Plainsboro, NJ) from a burr hole (Fig. 1).

The probe was placed into the brain tissue $2 \mathrm{~cm}$ deep from the dura. Applying NIRS, in addition to the parameters of ICP and brain temperature, the concentration of oxygenated and deoxygenated haemoglobin (Hboxy and Hbdeoxy), as well as CBF and cerebral blood volume (CBV), were measured.

(2) For conventional NIRS a plaster-based patch carrying optodes (one emitter, two detectors) was attached to the skin (NeMo Patch) (Fig. 2b). For spatially resolved spectroscopy (SRS) two detectors are placed at different distances from the laser source (distance from detector 1, $2 \mathrm{~cm}$; from detector 2, $4 \mathrm{~cm}$ ). Monte Carlo simulation of light propagation at 800 $\mathrm{nm}$ in a layered head model was performed with the same optode distances [1]. Two NIRS instruments, one connected to the probe, the other to the patch, specifically constructed for the measurement mode (NeMo Control Unit, NeMoDevices, Zurich, Switzerland) sources, the hardware for data collection and the software to analyse the NIRS data (Fig. 1).

Regular measurements were performed daily and repeated after 15 min under stable clinical conditions (unchanged ICP, mean arterial pressure and arterial partial pressure $\mathrm{CO}_{2}$ ). Central venous injections of $0.3 \mathrm{mg} / \mathrm{kg}$ body weight (bw) ICG were performed into a tube leading into an antecubital vein, followed by $10 \mathrm{ml}$ glucose $5 \%$ flush. ICG dye dilution curves obtained with the probe and patch were collected simultaneously and analysed for blood flow values (NeMoSystem ${ }^{\circledR}$, NeMoDevices, Zurich) based on published algorithms [10]. Measurement values are expressed in mean and standard deviation (SD). Measurement values are com- pared applying Wilcoxon matched pairs and calculating the correlation coefficient (Pearson; 2-tailed).

\section{Results}

In a first patient, 12 measurements in parallel with the probe and patch were performed. Mean measurement values for the mean transit time of ICG $\left(\mathrm{mtt}_{\mathrm{ICG}}\right)$, cerebral blood flow $(\mathrm{CBF})$ and cerebral blood volume $(\mathrm{CBV})$ obtained with the probe, as well as for the mean transit time of ICG (Emtt ICG $)$, blood flow (ECBF) and blood volume (ECBV) obtained with the

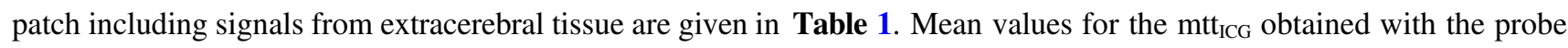
were significantly shorter compared with those obtained with the patch (for detector $1, p=0.002$; for detector $2, p=0.002$ ). Mean CBV obtained with the probe was significantly higher compared with the values obtained with the patch (for detector $1, p=0.002$; detector $2, p=0.002)$. The corresponding values for CBF were higher for the probe compared with those obtained with the patch (for detector $1, p=0.002$; for detector $2, p=0.002$ ). There were no significant differences between Emtt $t_{\text {ICG }}$ obtained with the patch detectors 1 and 2. However, ECBV and ECBF values obtained with detector 1 were slightly but significantly lower than those obtained with detector 2 (for ECBV, $p=0.003$; for ECBF, $p=0.004$ ).

The Monte Carlo simulations (Fig. 2a) show that, close to the source, light received by detector 2 is strongly reflected by the cerebrospinal fluid. At a larger distance from the source, most of the light that is received by detector 1 comes from the grey matter and a small portion from the white matter. CBF values obtained with the probe correlated significantly with blood flow values obtained with the patch (for CBF vs. ECBF detector 1, $r=0.72(p=0.008)$; ECBF detector $2, r=0.79(p=0.002))$.

Figure 3 shows dye dilution curves after ICG injection in optical density (OD) and in ICG concentration performed in parallel with the probe and patch. The maximum of the amplitude of the dye dilution curve measured with the probe occurs earlier and the amplitude is higher compared with NIRS measurements obtained with the patch through the scalp. 
Table 1 Mean measurement values for the mean transit time of of ICG $\left(\mathrm{mtt}_{\mathrm{ICG}}\right)$, cerebral blood flow (CBF) and cerebral blood volume (CBV obtained with the probe, as well as for the mean transit time of ICG (Emtt ICG ), blood flow (ECBF) and blood volume (ECBV) obtained with the patch including extracerebral tissu

\begin{tabular}{llll}
\hline & NeMo Probe $(n=12)$ & \multicolumn{2}{l}{ NeMo Patch $(n=12)$} \\
\cline { 3 - 3 } & & Detector 1 & Detector 2 \\
\hline Mtt $_{\text {ICG }}$ mean $(\mathrm{s})$ & $9.1 \pm 1.3$ & $15.9 \pm 3.2$ & $15.4 \pm 1.9$ \\
$\begin{array}{l}\text { Emtt } \\
\text { ICG mean }(\mathrm{s})\end{array}$ & & \\
CBV mean $(\mathrm{ml} / 100 \mathrm{~g})$ & $3.7 \pm 1.0$ & $1.3 \pm 0.3$ & $1.6 \pm 0.4$ \\
$\begin{array}{l}\text { ECBV mean }(\mathrm{ml} / 100 \mathrm{~g}) \\
\text { CBF mean }(\mathrm{ml} / 100 \mathrm{~g} / \mathrm{min})\end{array}$ & $24.8 \pm 9.1$ & & \\
ECBF mean $(\mathrm{ml} / 100 \mathrm{~g} / \mathrm{min})$ & & $5.1 \pm 1.8$ & $6.6 \pm 2.1$ \\
\hline
\end{tabular}
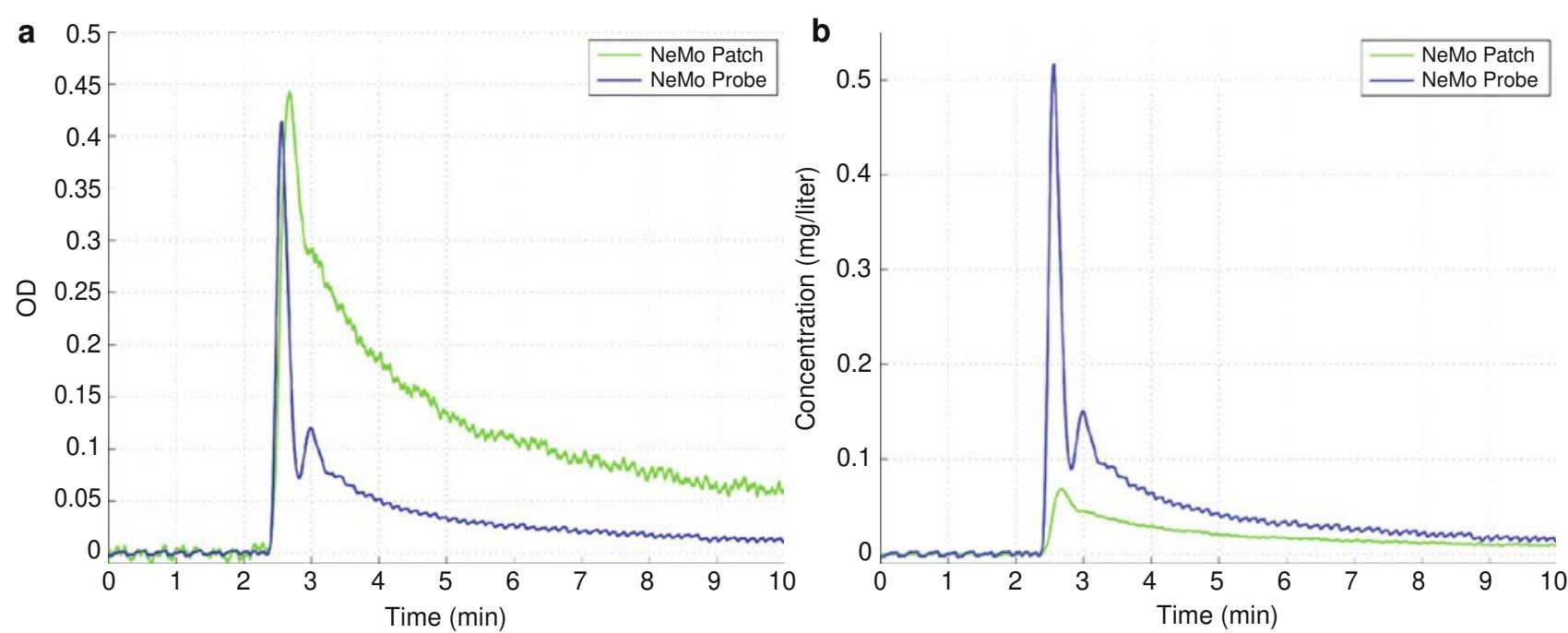

Fig. 3 Dye dilution curves after ICG injection in (a) optical density (OD) and (b) in ICG concentration in milligrams per litre (mg/l) performed in parallel with probe and patch

\section{Discussion}

Although NIRS seems to reflect significant changes in intra- cerebral vessels [11], it has not been demonstrated so far that these changes can be reliably distinguished from changes in extracerebral tissue [4, 15]. With the present study, for the first time, measurements in parallel with transcutaneous NIRS and a brain tissue probe with the tip in the white matter could be performed. The ECBF values obtained with conventional NIRS correlate significantly with CBF values obtained with the probe. The values for blood volume and flow from the NeMo Patch, however, were significantly lower compared with those from the NeMo Probe. ECBF values between 5.1 and $6.6 \mathrm{ml} / 100 \mathrm{~g} / \mathrm{min} \mathrm{measured} \mathrm{with}$ the patch correspond with blood flow values measured in the extracerebral tissue with the intravenous ${ }^{133}$ xenon dilution technique estimated to be $5-8 \mathrm{ml} / 100 \mathrm{~g} / \mathrm{min}$ [3]. Monte Carlo simulations, furthermore, show that the cumulative NIRS signal obtained by optodes over the skin is strongly influenced by extracerebral tissue [1, 14].

For SRS it has been suggested that applying one light emitter and two detectors allows subtraction of the extracerebral contamination from the cumulative NIRS signal obtained by optodes applied over the skin [6, 13]. Our measurement values obtained from the two detectors, 1 and 2 (4- and 2-cm apart from the light source) do not support this hypothesis. Inter-individual variability of anatomical struc- tures (bone thickness, extracerebral vasculature, liquid space, etc.) may restrict the reliability of SRS. Furthermore, the composition of the extracerebral tissue in neurosurgical patients will change during the illness course, especially after craniotomy.

Dye dilution curves after ICG injection performed in parallel with the NeMo Probe and the NeMo Patch showed different specific characteristics for probe and patch measurements, reflecting lower blood flow within extracerebral tissue. The maximum of the amplitude of the dye dilution curve measured with the probe typically occurs earlier reflecting a faster transit time of the dye. The amplitude of the curve obtained with the probe compared with the patch is higher, corresponding to a higher con- centration of ICG ICG in the brain tissue than in extracerebral structures. 


\section{Conclusions}

For the first time it was demonstrated that blood flow values obtained with conventional transcutaneous NIRS correlate significantly with absolute CBF values obtained directly within the brain tissue. With simultaneous measurements with the probe and patch, improved algorithms can be developed to quantify and subtract the contribution from extracerebral tissues from the signal obtained with conventional NIRS. This allows calculation of absolute values for CBF and oximetry data and definition of critical thresholds for ischemia not only for the NeMo Probe but also for the NeMo Patch.

\section{Source of Funding}

This project was supported by the Swiss Innovation Promotion Agency (CTI) project no. 13553.1PFFLM-LS and the Eurostars project no. E!6526 OPTO-BRAIN, as well as by the Velux foundation. NeMoDevices provided technical equipment for this clinical trial.

\section{Disclosures}

E. Keller, J. Froehlich, D. Baumann, M. Oberle and M. Muser have financial interests as founder, and staff members of NeMoDevices, AG. 


\section{References}

1. Boecklin C, Baumann D, Keller E, Froehlich J (2012) Determining light distribution in human head using 3D Monte Carlo simulations. Optical interactions with tissue and cells XXIII. Proc SPIE 8221:17

2. Colacino JM, Grubb B, Jobsis FF (1981) Infra-red technique for cerebral blood flow: comparison with xenon 133 clearance. Neurol Res 3:17-31

3. Friberg L, Kastrup J, Hansen M, Bulow J (1986) Cerebral effects of scalp cooling and extracerebral contribution to calculated blood flow values using the intravenous 133Xe technique. Scand J Clin Lab Invest 46:375-379

4. Germon TJ, Evans PD, Barnett NJ, Wall P, Manara AR, Nelson RJ (1995) Optode separation determines sensitivity of near infra- red spectroscopy to intra- and extracranial oxygenation changes. J Cereb Blood Flow Metab 15:S617

5. Germon TJ, Evans PD, Barnett NJ, Wall P, Manara AR, Nelson RJ (1999) Cerebral near infrared spectroscopy: emittordetector sepa- ration must be increased. Br J Anaesth 82:831-837

6. Germon TJ, Evans PE, Barnett NJ, Lewis TT, Wall P, Nelson RJ (1997) Changes in tissue oxyhemoglobin concentration mea- sured using multichannel near infrared spectroscopy during internal carotid angiography. J Neurol Neurosurg Psychiatry 63:660-664

7. Germon TJ, Kane NM, Manara AR, Nelson RJ (1994) Near- infrared spectroscopy in adults: effects of extracranial ischemia and intracranial hypoxia on estimation of cerebral oxygenation. Br J Anaesth 73:503-506

8. Hopton P, Walch TS, Lee A (1999) Measurement of cerebral blood volume using near-infrared spectroscopy and indocyanine green elimination. J Appl Physiol 87:1981-1987

9. Keller E, Froehlich J, Muroi C, Sikorski C, Muser M (2011) Neuromonitoring in intensive care: a new brain tissue probe for combined monitoring of intracranial pressure (ICP) cerebral blood flow (CBF) and oxygenation. Acta Neurochir Suppl 110:217-220

10. Keller E, Nadler A, Alkhadi H, Kollias S, Yonekawa Y, Niederer P (2003) Non invasive measurement of regional cerebral blood flow and regional cerebral blood volume by near infrared spectroscopy and indocyanine green dye dilution. Neuroimage 20:828-839

11. Kuebler WM, Sckell A, Habler O, Kleen M, Kuhnle GEH, Welte M, Messmer K, Goetz AE (1998) Noninvasive measurement of regional cerebral blood flow by near-infrared spectroscopy and indocyanine green. J Cereb Blood Flow Metab 18:445-456

12. McCormick PW, Stewart M, Goetting MG, Dujovny M (1991) Noninvasive cerebral optical spectroscopy for monitoring cerebral oxygen delivery and hemodynamics. Crit Care Med 19:89-97

13. Miwa M, Ueda Y, Chance B (1995) Development of time resolved spectroscopy system for quantitative non-invasive tissue measure- ment. Proc SPIE 2389:142-149

14. Mudra R, Nadler A, Niederer P, Keller E (2006) Analysis of near- infrared spectroscopy and indocyanine green dye dilution with Monte Carlo simulation of light propagation in the adult brain. J Biomed Opt 11:044009-044013

15. Owen-Reece H, Elwell CE, Wyatt JS, Delpy DT (1996) The effect of scalp ischemia on measurement of cerebral blood volume by near-infrared spectroscopy. Physiol Meas 17:279-286

16. Schwarz G, Litscher G, Kleinert R, Jobstmann R (1996) Cerebral oximetry in dead subjects. J Neurosurg Anesthesiol 8:189-193 